\title{
Modelling of Buried Object Detection Using Thermography
}

\author{
by Ivanka Boras, Marina Malinovec, Josip Stepanic jr. and Srecko Svaic
}

University of Zagreb, Faculty of Mechanical Engineering and Naval Architecture,

I. Lucica 5, HR-10000 Zagreb, Croatia, T: ++385 16168 222, F: ++385 16156 940,

Es:ivanka.boras@fsb.hr,marina.malinovec@fsb.hr,josip.j.stepanic@fsb.hr,srecko.svaic@fsb.hr

\section{Abstract}

In order to evaluate a possibility for buried object detection using active IR the laboratory set-up was made. The experimental system consists of a box containing two different soil types. In soils, objects of different dimensions are buried. The surface temperature distribution is measured using thermography. Additionally, the soil temperature depth profile and soil humidity are measured. Using the developed mathematical model and measured temperature distribution the possibility for buried object detection and identification are discussed.

The objective of the work is to try to develop applicable method for precise buried object position determination. Results obtained are discussed from the point of view of possible application of the method for buried antipersonnel landmines detection in the humanitarian demining.

\section{Introduction}

In a thermography one detects differences in infrared radiation intensity emitted from the surface of an object. The differences are caused by the different heat content of the object or its various parts, additionally influenced by the surface emissivity characteristics. When a nonhomogeneous structure, having different thermal characteristics, initially being in thermal equilibrium with its surrounding, is exposed to heat stimulation the temperature difference occurs in the structure as well as on their visible surfaces [1]. One differs the active and passive thermography.

Listed characteristics made thermography a promising method for buried objects detection. The already existing applications of thermography for that purpose are found in a variety of civil and mechanical engineering operations, as well as in other fields.

In the last several years the field of thermography's applications has included the detection of the buried landmines [2-5]. The necessity for localisation and identification of landmines has arisen during last decade. The estimate is that there are about 80 millions landmines buried world-wide. The present status of various detection methods is such that in the field pyrotechnicians use prodders, specially trained dogs, metal detectors and occasionally ground penetrating radars. The methods listed are contact or almost contact non-destructive testing methods, the usage of which is in the demining accompanied with high risk level, large duration and expenses [6-8]. Other detection methods are not reliable enough or the conditions for their applicability are not clear [6]. These reasons motivated work on development of reliable, non-contact, safe and efficient antipersonnel landmines detection method [6, 9].

A considerable work has been done regarding evaluation of the thermography's buried landmines detection capability using non-polarised infrared radiation [2-5, 7, 9]. In the work of Pregowski et al. the applications of passive thermography has been thoroughly evaluated in a combined theoretical and experimental work. Somewhat conversely to that Svaic et al. concentrated on the active thermography applications. The present status of these approaches is that each of them provides an operator with important pieces of information about soil subsurface layer. Both methods are important for full exploitation of the thermography in buried landmines detection. The complementarity of the methods lies in the duration and energy consumption of the detection. In the applications that use passive thermography the energy for thermal contrast induction is provided through solar radiation. In cases this radiation is intense enough heating costs of the method are virtually absent. The duration of a particular area detection is determined with the diurnal and seasonal cycles. However, in cases when solar radiation is not intense 
enough, the passive thermography is not applicable. On the other side, active thermography applications are accompanied with relatively large heating costs but are independent of solar radiation intensity. Both methods are further limited, and in some situations made impossible by soil characteristics, vegetation layer properties and local-in-space-and-time climate conditions.

The heat flux provided by heaters used in active thermography can be much larger then solar heat flux. However, artificial heat sources of large enough heat flux are non-efficient because of relatively large energy consumption and additional equipment required. From combination of requirements for relatively small energy consumption and mine contaminated regions' characteristics one concludes that there are regions where thermography can be an efficient APL detection method. The examples of this type of regions are dry, vegetationless soils. On the contrary, in wet soils, covered with dense vegetation layer at the present level of development the thermography is non-applicable. The problem of precise border of thermography's applicability is by no means a solved one [9].

In this article we work on setting the reliable and easy determinable measure of the applicability of the active thermography for the buried objects detection. In order to accomplish the given task, the idealised conditions were prepared as the starting point. They include a combination of homogeneous, vegetationless and planar surface soil layer containing homogeneous objects of relatively simple geometry. Additionally, reliable theoretical model enables one to further simplify the needed measure evaluation. Because of that, we set the theoretical model that simulates the process of active thermography applications on buried objects detection.

In the next section the experimental part of the work is described, while in the third section the numerical simulation is given. The results obtained are discussed in the fourth section, while the most important conclusions are given in the fifth section.

\section{Experiment}

\subsection{Introduction}

The estimation of possible APL detection method based on thermography was experimentally performed at the Faculty of Mechanical Engineering and Naval Architecture, University of Zagreb in Croatia by joint efforts of Laboratory for Applied Thermodynamics and Laboratory of NonDestructive Testing.

The experimental set-up, shown on figure 1a, consists of the thermovision camera AGA 680 Standard cooled by liquid nitrogen, the corresponding monitors and computer manipulated system for measurements of temperatures in different positions. Temperatures were measured using thermocouples. The box visible on figure $1 \mathrm{a}$ is divided in two parts, that were during the experiment each filled with dry sand of particular grain size. The measurements presented here were performed on the part filled with finer grain sand.

The objects used in measurements were homogeneous cylinders made of polyethylene, having same diameter of $30 \mathrm{~mm}$, but differing in heights. Further in the text, numbers 1, 2, 3, 4 and 5 denote cylinders of heights $5 \mathrm{~mm}, 10 \mathrm{~mm}, 20 \mathrm{~mm}, 30 \mathrm{~mm}$ and $60 \mathrm{~mm}$, respectively. They were buried as shown on figure $1 \mathrm{~b}$, in a depth of $8 \mathrm{~mm}$.

Thermocouples were put on upper and lower planes of cylinders 2 and 4 . This way one ends with a moderate quantity of data about the heat exchange processes that, at the same time, enable one to fully reproduce the processes. Additionally, surface temperature was measured to enable sand surface temperature quantification using thermogram.

Sand surface was heated using infrared heater. The heating with constant heat flux lasted determined time after what the heating was turned off. Temperature measurements included the phases of the heating and subsequent cooling.

\subsection{Results}

Results of the experiment are shown for described configurations of objects.

Heating by infrared heater with heat flux of $15000 \mathrm{~W} \mathrm{~m}^{-2}$ lasted at least $300 \mathrm{~s}$. Temperature was recorded during heating and additional relatively long time during subsequent cooling. Here, 
results are shown when heating lasted $300 \mathrm{~s}$ and $600 \mathrm{~s}$. The interval of subsequent temperature recording lasted about $3000 \mathrm{~s}$.

Temperatures of centres of upper surfaces of objects and centres of lower surfaces of objects 2 and 4 were recorded. The surface temperature means temperature of soil 1-2 $\mathrm{mm}$ under soil surface. It was measured between the objects 3 and 4 approximately between them.

Graphs of time dependence of temperature for objects' configurations used is presented on figure 2. Surface's thermograms for different heating durations, recorded in various moments during the system cooling are shown on figure 3.

\section{Numerical Analysis in Buried Object Detection Using Thermography}

\subsection{Introduction}

The numerical analysis of heat transfer is based on the application of control volumes method [1].

The control volume's net is set so that regions of higher thermal gradients in the model are accompanied with locally denser net. The larger number of control volumes in these regions enables better information about temperature distribution. Buried objects are treated as nonhomogenities in the structure of primary material, i.e. soil.

Numerical solving of tridimensional, nonstationary heat conduction requires initial and boundary conditions, that are taken from the experimental part of the work, figure 4.

In the initial situation the temperature is uniform and known.

\subsection{Results}

Values of the parameters describing sand are $\lambda=0,582 \mathrm{~W} \cdot \mathrm{m}^{-1} \cdot \mathrm{K}^{-1}, \rho=1650 \mathrm{~kg} \cdot \mathrm{m}^{-3}$ and $c=712$ $\mathrm{J} \cdot \mathrm{kg}^{-1} \cdot \mathrm{K}^{-1}$, while values for the polyethylene were $\lambda=0,45 \mathrm{~W} \cdot \mathrm{m}^{-1} \cdot \mathrm{K}^{-1}, \rho=940 \mathrm{~kg} \cdot \mathrm{m}^{-3}, c=1780 \mathrm{~J} \cdot \mathrm{kg}^{-1} \cdot \mathrm{K}^{-1}$.

Initial temperature was taken to be $26^{\circ} \mathrm{C}$ on the lower sand part and $27^{\circ} \mathrm{C}$ on sand surface and in air. Sand surface emmisivity was taken as $\varepsilon=0,96$. Heating lasted for $300 \mathrm{~s}$.

The non-structured net consisted of 28512 elementary volumes. Each object's perpendicular cross section covered 24 elementary volumes. Time step in the numerical integration was equal to $0,2 \mathrm{~s}$.

The time dependence of temperature for various objects is shown on figure $5 \mathrm{a}$, and contour plot of surface temperature field on figure $5 \mathrm{~b}$.

\section{Discussion}

The results obtained show a number of interconnected heat transfers between the heater and soil, heater and environment, and soil and buried objects. These transfers could be understood using figures 2, 3 and $5 \mathrm{a}$. Discussion is given for the configuration of objects shown on figure $1 \mathrm{~b}$.

Large temperature differences achieved were possible because the dry and vegetationless soil was used. With the larger water content, temperatures achieved would have been lower for given heating time, or duration of the experiment longer for given temperature difference.

During heating, and some time after it was stopped, the surface temperature was the highest among measured ones, reflecting the initial heat accumulation in the thin sand layer over the objects. After that, as heat was distributed to lower soil layers and objects, the objects upper surfaces' temperatures become the highest among measured ones. According to simulation, the object 1 upper surface temperature was even higher. Hence, in that interval the objects figure as heat reservoirs dissipating heat in the environment. Two additional consequences of the heat conduction is that lower parts of the system (sand and objects) achieve their maximal temperatures later than upper parts and that those maximas are lower than for upper parts.

On the thermograms, figure 3 , a difference in the emitted heat is seen. This is a consequence of a different heat content accumulated during the heating phase in objects. Because of the relatively small differences in heat characteristics of sand and polyethylene (see section 3.2.), there was no crucial differences in the amounts of heat conducted through the objects and through 
the sand. This is seen from the approximately equal values of objects' 2 and 4 upper surface temperatures. On the other hand, that means that the sand remained effectively infinitely deep during the experiment.

Energy consumption in the experiment was $2,5 \mathrm{kWh} \cdot \mathrm{m}^{-2}$. Detection duration was determined effectively to be 40 min measured from the beginning of the heating.

This model may be appropriately scaled and therefore describe a class of geometrically similar models with buried objects of various dimensions. If one scale all the quantities encountered according to

$$
\left\{\begin{array}{cc}
T, \phi \\
\lambda, & \rho, c \\
r, & t
\end{array}\right\} \rightarrow\left\{\begin{array}{c}
\mu_{T} T, \mu_{\phi} \phi \\
\mu_{\lambda} \lambda, \mu_{\rho} \rho, \mu_{c} c \\
\mu_{r} r, \mu_{t} t
\end{array}\right\},
$$

then from the solution $T(x, y, z, t, \phi)_{\lambda, \rho, c}$ one obtains directly the solution $\mu_{\top} T\left(\mu_{\mathrm{r}} x, \mu_{\mathrm{r}} y, \mu_{\mathrm{r}} z, \mu_{\mathrm{t}} t\right.$; $\left.\mu_{\phi} \phi\right)_{\mu \lambda, \mu \rho, \mu c}$ for the similar problem without changes in heating source as long as $\lambda, \rho$ and $c$ do not depend on infrared radiation wavelength. Because of the simplicity all different soil's and objects' characteristics were written without additional indexes. This scaling property means that if one wants to compare two experiments differing only in the geometry $\left(\mu_{\lambda}=\mu_{\mathrm{p}}=\mu_{\mathrm{c}}=1, \mu_{\mathrm{t}}=\mu_{\mathrm{r}}{ }^{2}\right.$ and $\left.\mu_{\phi}=\mu_{\mathrm{T}} / \mu_{\mathrm{t}}\right)$ there are two independent scaling factors, $\mu_{\top}$ and $\mu_{\mathrm{t}}$.

\section{Conclusions}

Using the active thermography and nonpolarised infrared radiation, on a given set of polymeric objects put in homogeneous, dry and vegetationless soil, the non-stationary thermal field was realised. The values of temperature in several characteristic points of the system was measured. Using the numerical simulation the thermal field was reproduced. This field is rather sensitive on the physical properties of objects. The existing difference in physical properties means that in the relatively large time interval of heating and subsequent cooling of heated region large enough thermal gradients and temperature differences are obtained. On the basis of that, the detection and differentiation of the buried objects is in some cases possible.

However, in non-homogeneous soils the possibility of buried objects detection and differentiation becomes questionable. This possibility is additionally influenced by buried objects' shapes.

\section{REFERENCES}

[1] Andrassy (M.), Svaic (S.) and Boras (I.). - Development of Applied Thermographic Methods. International Congress SITHOK 4, Maribor, Proceedings, 2000., pp. 48.1-48.7,

[2] Pregowski (P.) and Swiderski (W.). - Application of IR Thermography for the Detection of Buried Mines - Prediction and Procedures, International Conference MATEST '99, Cavtat, Proceedings, 1999., pp. 227-232,

[3] Pregowski (P.), Swiderski (W.) and Walczak (R.). - Surface and volume effects in thermal signatures of buried mines: experiment and modeling. QIRT '98, Lodz, Proceedings, 1998., pp. 233-238,

[4] Georgson (M.) et al. - Mine Detection Using Infrared Imaging Technique. International Conference MINE'99, Firenze, Proceedings, 1999., pp. 66-71,

[5] Schachne (M.) et. al. - Mine Detection by Means of Dynamic Thermography: Simulation and Experiments. IEE 2nd International Conference on the Detection of Abandoned Landmines '98, Edinburgh, Proceedings, 1998., pp. 124-128,

[6] Krstelj (V.) and Stepanic jr. (J.). - Humanitarian de-mining detection equipment and working group for antipersonnel landmines detection. INSIGHT, Vol. 42(3), 2000., pp. 187-190, 
[7] Bruschini (C.) and Gros (B.). - A Survey of Current Sensor Technology Research for the Detection of Landmines. International Workshop SUSDEM '97, Zagreb, Proceedings, 1999., pp. 6.18-6.27,

[8] Krstelj, (V.) and Stepanic, jr. (J.). - Non-Destructive Testing in Antipersonnel Landmine Detection, International Conference MATEST '99, Cavtat, Proceedings, 1999., pp. 109-115,

[9] Krstelj (V.), Svaic (S.), Stepanic jr. (J.) and Malinovec (M.). - NDT Methods in Landmines Detection. International Conference Defektoskopie '99, Hradec Kralove, Proceedings, 1999., pp. 251-256,

[10] Krstelj (V.), Stepanic jr. (J.) and Leljak, (I.). - Evaluation and Certification of Humanitarian Demining Detection Equipment, Journal of Mine Action 4.1, Spring 2000., pp. 60-61.

a)

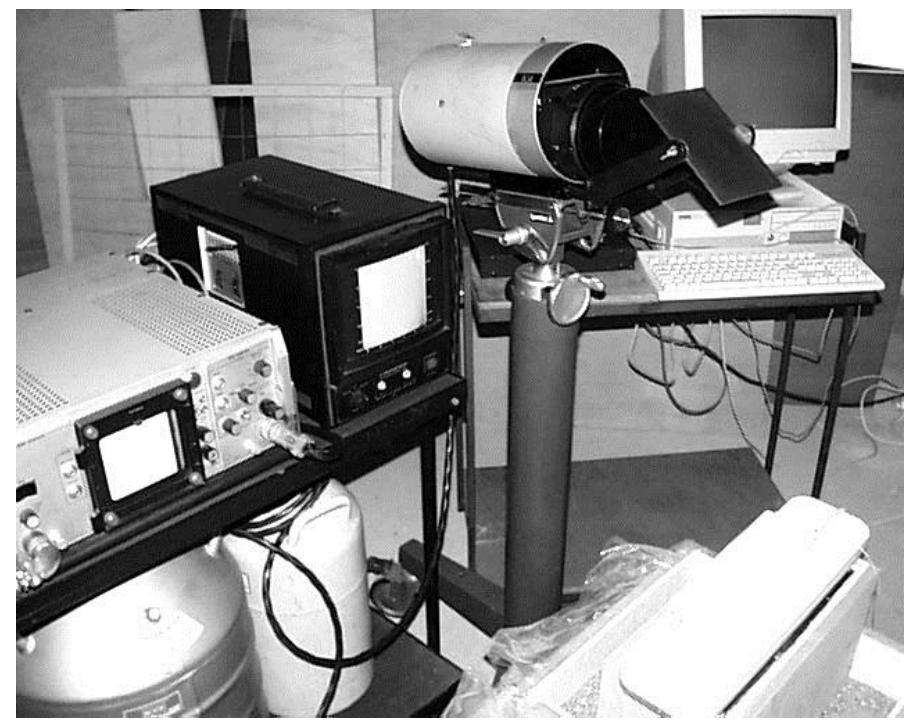

b)
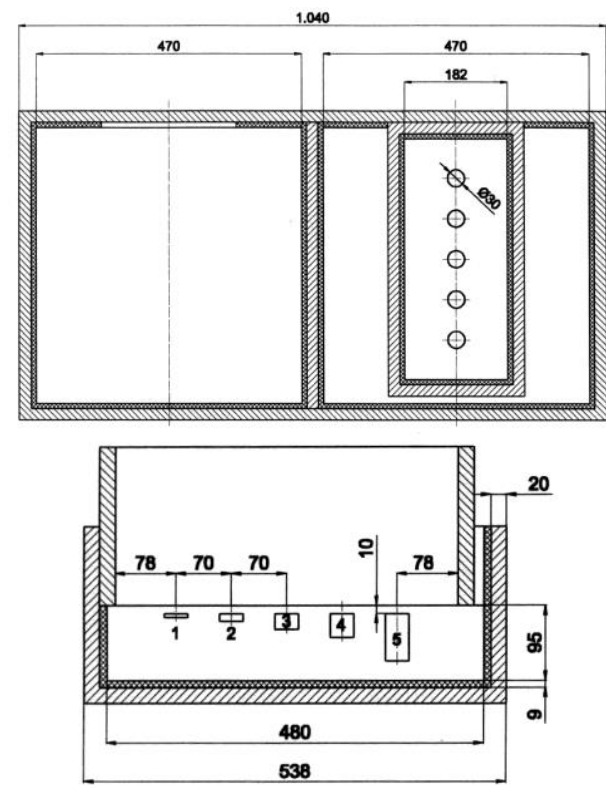

Fig. 1. a) Experimental set-up. A heater and a part of the box filled with a sand are seen in the lower right part of the figure. b) Schematic representations of the box with sand and buried objects.

The horizontal distance between the neighbouring objects is slightly bigger than the objects' diameter. Temperatures were measured in the centres of the upper and lower bases of objects 2 and 4 . Surface temperature was measured as well.
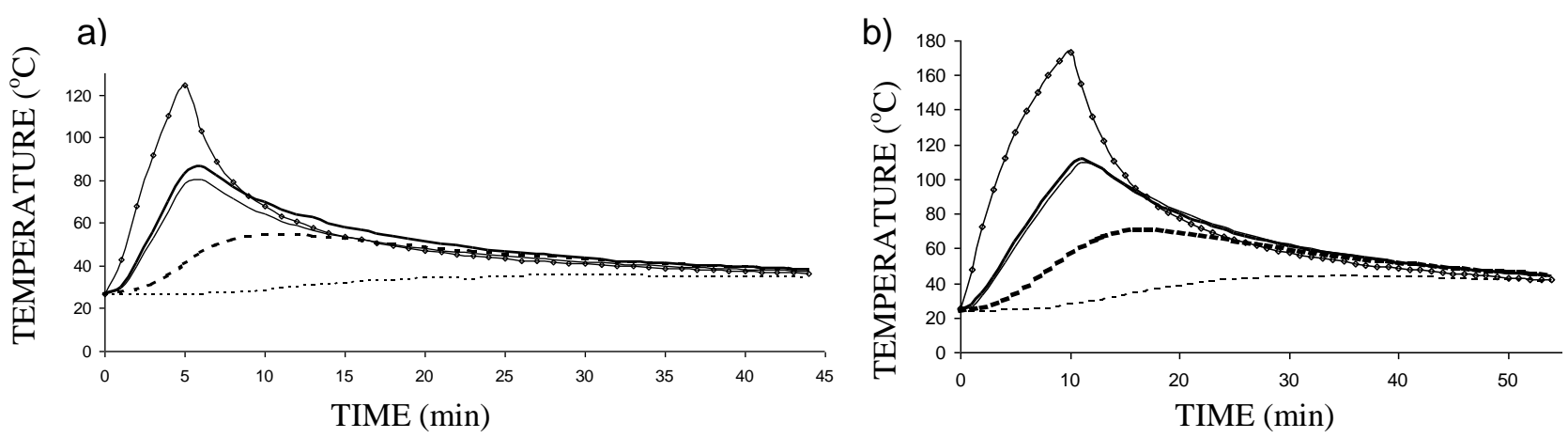

Figure 2. Measured time dependence of temperature for heating duration of a) $300 \mathrm{~s}, \mathrm{~b}) 600 \mathrm{~s}$. Upper (lower) base's temperatures are represented with regular (dashed) lines. Object 2's (4's) temperatures are represented with bold (non-bold) lines. Surface temperatures represent lines with romps. 
a)

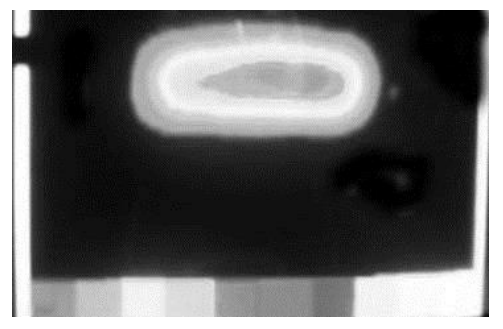

b)

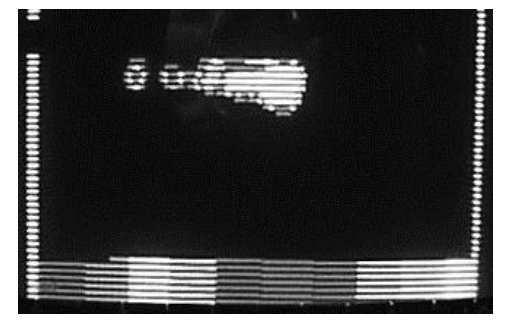

c)

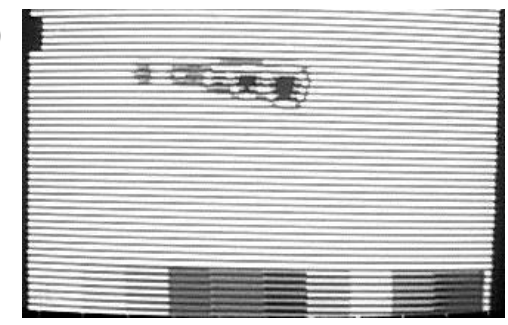

Figure 3. Thermograms of soil surfaces above the objects in configuration shown on fig. 1b. a) normal mode, heating duration $300 \mathrm{~s}$, thermogram recorded $120 \mathrm{~s}$ after heating was stopped, b) normal mode, heating duration $600 \mathrm{~s}$, thermogram recorded $120 \mathrm{~s}$ after heating was stopped, c) inverse mode, heating duration $600 \mathrm{~s}$, thermogram recorded $180 \mathrm{~s}$ after heating was stopped.

Object 1 is the farthest on the right.

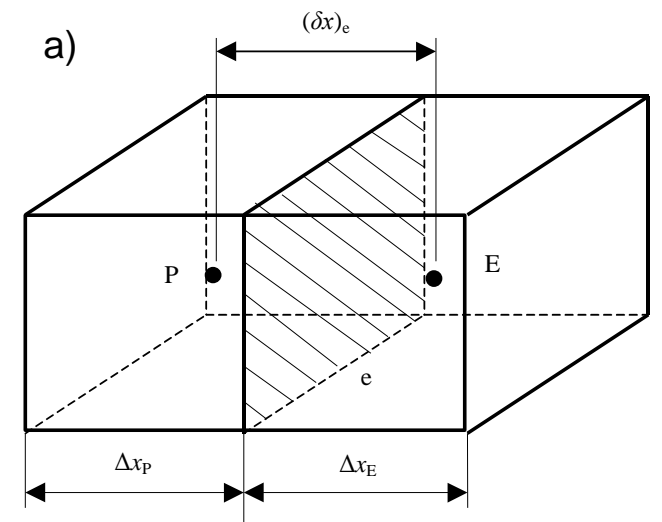

b)

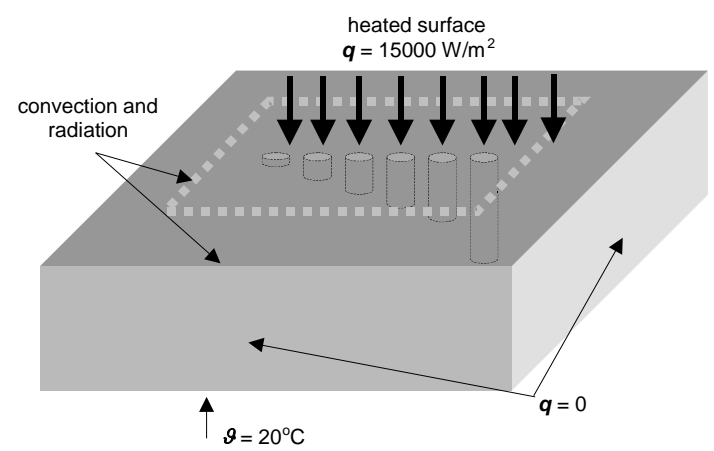

Fig. 4. a) Model for calculating thermal conductivity, b) imposed boundary conditions

a)

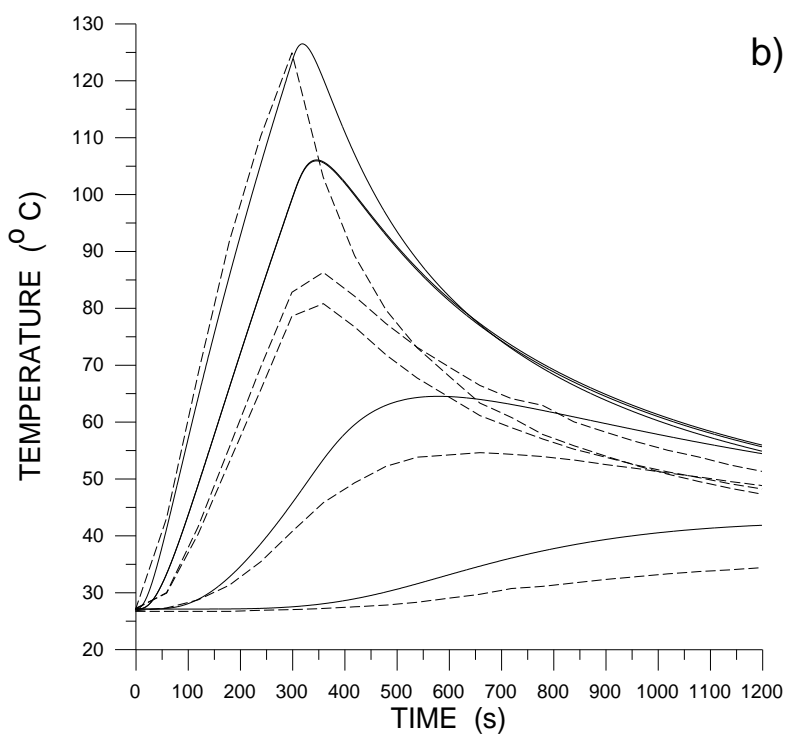

b)

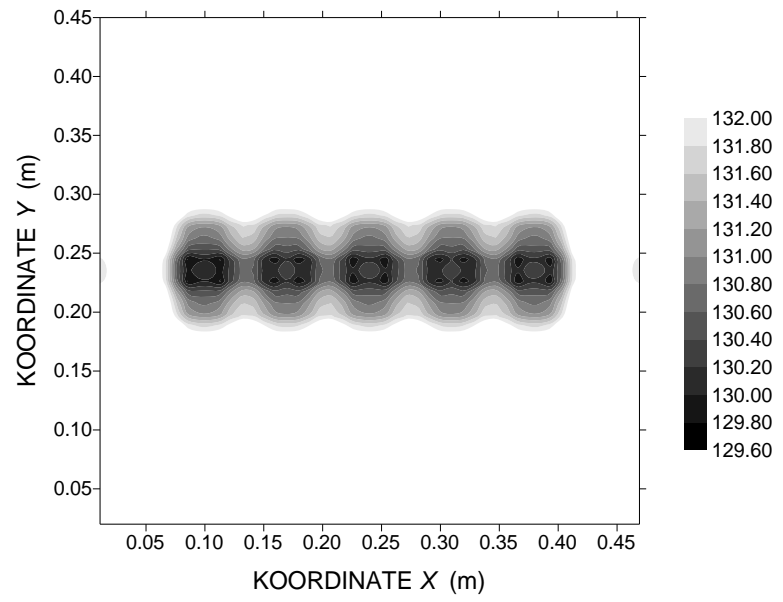

Figure 5. For $300 \mathrm{~s}$ heating duration a) calculated (calc.) and measured (meas.) temperatures' time dependence, b) contour plot of surface temperature field $240 \mathrm{~s}$ after heating was stopped. On figure $5 \mathrm{a}$ various lines, listed according to their maxima from the highest are temperatures at surface calc., surface meas., objects 2 and 4 upper bases calc., object 2 upper base meas., object 4 upper base meas., object 2 lower base meas., object 2 lower base calc., object 4 lower base meas. and object 4 lower base calc. The soil and objects parameters used in calculations somewhat differed from that found in the experiment. 